\title{
Effect of asiaticoside on endothelial cells in hypoxia-induced pulmonary hypertension
}

\author{
XIAOBING WANG $^{1 *}$, XUELI CAI $^{2 *}$, WU WANG $^{3 *}$, YI JIN $^{1}$, MAYUN CHEN $^{4}$, \\ XIAOYING HUANG ${ }^{4}$, XIAOCHUN ZHU ${ }^{1}$ and LIANGXING WANG ${ }^{3}$
}

\begin{abstract}
Departments of ${ }^{1}$ Rheumalogy and ${ }^{2}$ Cardiology, The First Affiliated Hospital of Wenzhou Medical University;
${ }^{3}$ Key Laboratory of Laboratory Medicine, Ministry of Education of China, School of Laboratory Medicine and Life Sciences, Wenzhou Medical University; ${ }^{4}$ Key Laboratory of Heart and Lung, Respiratory Department,

The First Affiliated Hospital of Wenzhou Medical University, Wenzhou, Zhejiang 325035, P.R. China
\end{abstract}

Received December 20, 2016; Accepted November 1, 2017

DOI: $10.3892 / \mathrm{mmr} .2017 .8254$

\begin{abstract}
Pulmonary hypertension (PH) is a chronic progressive disease with limited treatment options. The exact etiology and pathogenesis of $\mathrm{PH}$ remain to be elucidated, however there is novel evidence that implicates abnormal endothelial cells (ECs) apoptosis and dysfunction of ECs to be involved in the initiation of PH. Asiaticoside (AS) is a saponin monomer extracted from a medicinal plant called Centella asiatica, which had a preventing effect of hypoxia-induced pulmonary hypertension (hypoxic $\mathrm{PH}$ ) by blocking transforming growth factor- $\beta 1 /$ SMAD family member $2 / 3$ signaling in our previous study. The present study demonstrated that AS can prevent the development of hypoxic $\mathrm{PH}$ and reverse the established hypoxic $\mathrm{PH}$. AS may activate the nitric oxide (NO)-mediated signals by enhancing the phosphorylation of serine/threonine-specific protein kinase/eNOS, thus promoting NO production, and prevent ECs from hypoxia-induced apoptosis. All these findings imply that AS may be a potential therapeutic option for hypoxic PH patients due to its effect on the vitality and function of endothelial cells.
\end{abstract}

Correspondence to: Dr Liangxing Wang, Key Laboratory of Laboratory Medicine, Ministry of Education of China, School of Laboratory Medicine and Life Sciences, Wenzhou Medical University, 1 Xuefu North Road, Wenzhou, Zhejiang 325035, P.R. China

E-mail:wzwxbtgzy@163.com

Dr Xiaochun Zhu, Department of Rheumalogy, The First Affiliated Hospital of Wenzhou Medical University, 1 Xuefu North Road, Wenzhou, Zhejiang 325035, P.R. China

E-mail: gale820907@163.com

"Contributed equally

Key words: pulmonary hypertension, endothelia cells, asiaticoside, nitric oxide, apoptosis

\section{Introduction}

Pulmonary hypertension ( $\mathrm{PH})$ is a chronic progressive disease characterized by increased pulmonary vascular resistance and pulmonary vasculature remodeling. Although $\mathrm{PH}$ has a high incidence of mortality (1), the exact etiology and pathogenesis remain to be elucidated. There is increasing evidence that implicates abnormal endothelial cells (ECs) apoptosis and ECs dysfunction to be involved in the initiation and development of PH $(2,3)$. ECs isolated from the idiopathic pulmonary arterial hypertension patients had disordered growth features (4). However, enhanced EC growth and survival, associated with reduced EC apoptosis may prevent the development of $\mathrm{PH}$ induced by monocrotaline $(5,6)$. Additionally, dysfunction of ECs may be a consistent marker of $\mathrm{PH}$ in rodents and humans (4). In hypoxic PH, the dysfunction of ECs was believed to lead to the reduction in the endothelium-derived nitric oxide (NO) production (7).

NO, working as a potent vasodilator has become an important therapeutic target for hypoxic PH. Endogenous $\mathrm{NO}$ is produced by nitric oxide synthase (NOS), which has been efficiently phosphorylated by serine/threonine-specific protein kinase (Akt) activation $(8,9)$. However, the expression of endothelial NOS (eNOS) is reduced (10), whilst the production of endogenous NOS inhibitors, such as asymmetrical dimethylarginine (ADMA) and symmetrical dimethylarginine (SDMA) is enhanced in PH (11). These findings indicated that protecting EC from abnormal apoptosis and normalization of the dysregulated NO-mediated signal in ECs may be potential therapeutic strategies in patients with hypoxic $\mathrm{PH}$.

Asiaticoside (AS) is a saponin monomer extracted from a medicinal plant termed Centella asiatica. It has been previously documented to have multiple biological effects, such as antioxidant (12), anti-inflammatory (13), anti-hepatofibrotic (14) and acting as a neuroprotector against transient cerebral ischemia and reperfusion (15). However, further investigation that highlights its protective effects on ECs is required. Our previous study revealed rudimentary understanding, that AS may prevent the development of $\mathrm{PH}$ by attenuating pulmonary cardiovascular remodeling in hypoxia-induced $\mathrm{PAH}$ rats, which may be mediated by blocking the hypoxia-induced 
over activity of transforming growth factor (TGF)- $\beta 1 / \mathrm{SMAD}$ family member (SMAD) 2/3 signaling (16). However, whether AS may attenuate established hypoxic $\mathrm{PH}$ and its effects on the vitality and function of ECs remains to be determined.

Therefore, the present study compared the effect of AS on hypoxic $\mathrm{PH}$ rats with different treatment strategies. Subsequently, the effect of AS on EC function was examined by evaluation of $\mathrm{NO}$ production and AKT/eNOS activation in vivo and in vitro and investigated its effects on EC survival and apoptosis in vitro. The present study indicated that AS may prevent the development of hypoxic PH and attenuate established hypoxic $\mathrm{PH}$, which may be primarily due to the enhanced NO-mediated signal and the reduced apoptosis of EC under hypoxia.

\section{Materials and methods}

Animal experimental protocols. Animal experiments were approved by the Institutional Animal Ethics Committee for Experimentation on Animals of Wenzhou Medical University (Wenzhou, China) and followed the National Institutes of Health Guide for the Care and Use of Laboratory Animals. A total of 40 Adult male Sprague-Dawley rats with a body weight of 180-200 g (SLAC Laboratory Animal Co., Ltd., Shanghai, China) were used in experiments and they were kept at $25^{\circ} \mathrm{C}, 21 \% \mathrm{O}_{2}$, a $12 \mathrm{~h} / 12 \mathrm{~h}$ light/dark cycle and free access to food and water. The rats were then separated randomly into four groups, and each group contained 10 rats: i) Control rats raised in normoxia for 4 weeks (Nox); ii) control rats raised in hypoxia for 4 weeks $(\mathrm{Hx})$; iii) rats raised in hypoxia and received AS (diluted with normal saline; Sigma-Aldrich; Merck Millipore, Darmstadt, Germany) from the same day for 4 weeks (HP); and iv) rats raised in hypoxia for 2 weeks, then received the 4-week AS treatment, i.e., continued another 2 weeks' treatment after finishing the 4-week hypoxia exposure (HT). Hypoxia-induced PH was developed by keeping rats in a sealed, but ventilated hypoxic chamber $\left(9 \% \mathrm{O}_{2}\right.$, YPC-160D; Changsha Huaxiao Electronic Technology Co., Ltd., Changsha China) as previously described (17). The treatment groups, HP and HT group received AS (50 mg/ $\mathrm{kg}$ ), while Nox and Hx groups received vehicle (normal saline, 1.5-2 ml) administration as control. AS and vehicle were administered daily through intragastric administration.

Examination of mean pulmonary artery pressure and right ventricular hypertrophy. After treatment, rats were weighed and received sodium pentobarbital (Sigma-Aldrich; Merck Millipore) anesthetization by intraperitoneal injection (35 mg/kg). Invasive hemodynamic measurements, including mean pulmonary arterial pressure (mPAP) and mean carotid arterial pressure (mCAP) were examined as described previously (18). Following the measurement, the right ventricular (RV) wall was separated from the left ventricular (LV) wall, and the interventricular septum (S) and weighed. An index of right ventricular hypertrophy (RVH) was calculated by right ventricle to left ventricle plus septum ratio.

Morphometric analysis of pulmonary artery. Hematoxylin and eosin (H\&E) staining (hematoxylin for $5 \mathrm{~min}$ and eosin for $30 \mathrm{sec}$ at $25^{\circ} \mathrm{C}$ ) was performed and the images ( $\mathrm{x} 400$ magnification) of the lung tissue (5- $\mu \mathrm{m}$-thick sections) and pulmonary arterioles were captured with a microscope digital camera (BX51 microscope; Olympus Corporation, Tokyo, Japan). The percent of medial wall thickness was determined by Image-Pro Plus 6.0 as previously described (19). A small part of flesh lung tissue was maintained in glutaraldehyde. The 3- $\mu$ m-thick sections of each sample were fixed first with $25 \%$ glutaraldehyde $2 \mathrm{~h}$ at $4^{\circ} \mathrm{C}$, then incubated with $1 \%$ osmic acid $1 \mathrm{~h}$ at $25^{\circ} \mathrm{C}$, stained with uranyl acetate for $1 \mathrm{~h}$ and lead citrate for $1 \mathrm{~h}$ at $25^{\circ} \mathrm{C}$, embedded with ethoxyline resin $24 \mathrm{~h}$ at $60^{\circ} \mathrm{C}$, and then observed under transmission electron microscope detection (H-600 transmission electron microscope; Hitachi, Ltd., Tokyo, Japan).

Measurement of endothelin (ET)-1, prostacyclin (PGI2), cyclic guanosinc monophosphate (cGMP) and NO in vivo. The $1 \mathrm{ml}$ blood samples were taken from each rat's heart after hemodynamic measurements to obtain serum at a volume of $150 \mathrm{ml}$, which was centrifuged at $200 \mathrm{xg}, 8 \mathrm{~min}$ at $25^{\circ} \mathrm{C}$. The levels of ET-1 in the serum of rats were determined by enzyme-linked immunosorbent assay (ELISA) kit (cat. no. ABP52878; Abcam, Cambridge,UK). PGI2 concentration was also tested by ELISA kit (cat. no. MBS266717; MyBioSource, Inc., San Diego, CA, USA). cGMP levels in lung tissue was quantified by the cGMP Direct Immunoassay kit (cat. no. 581022-96; Abcam). The level of NO measured by quantitating total nitrate/nitrite using nitric oxide colorimetric assay kit (cat. no. K205-100; BioVision, Inc., Milpitas, CA, USA). All the procedures were completed following to the manufacturer's instructions. Data were quantified using a standard curve of known concentrations. Each sample was evaluated in triplicate.

Cell treatment and NO production of HPAECs. According to the results of cell viability, HPAECs were divided into the following four groups: i) Nox, cells were cultured under normoxia $\left(21 \% \mathrm{O}_{2}, 5 \% \mathrm{CO}_{2}\right)$; ii) $\mathrm{H} 0$, cells were cultured under hypoxia $\left(5 \% \mathrm{O}_{2}, 5 \% \mathrm{CO}_{2}\right)$; iii) $\mathrm{H} 50$, cells were cultured with AS (50 $\mu \mathrm{g} / \mathrm{ml})$ under hypoxia; iv) HL, cells were cultured with AS $(50 \mu \mathrm{g} / \mathrm{ml})$ and LY294002 $(20 \mu \mathrm{mol} / 1)$ under hypoxia, 3 wells were used per group. All cells were cultured at $37^{\circ} \mathrm{C}$ for $24 \mathrm{~h}$. The production of NO in HPAECs was quantified by the same colorimetric assay used for the detection of NO levels in rat serum as aforementioned.

Apoptosis detection. After treatment, the cells were detected by the terminal deoxynucleotidyl-transferase-mediated dUTP nick end labeling (TUNEL) assay kit (Roche Applied Science, Penzberg, Germany). A total of four groups were fixed of air-dried cell samples with a freshly prepared fixation solution for $1 \mathrm{~h}$ at $25^{\circ} \mathrm{C}$, then incubated with blocking solution and permeabilization solution sequentially for $10 \mathrm{~min}$ and 2 min at $25^{\circ} \mathrm{C}$ respectively after rinsing slides with PBS in between. The TUNEL reaction mixture was prepared and $50 \mu \mathrm{l}$ was added on sample and incubate for $60 \mathrm{~min}$ at $37^{\circ} \mathrm{C}$. Samples at last analyzed in a drop of PBS under a fluorescence microscope (CX21FS1; Olympus) with the wavelength of $550 \mathrm{~nm}$. TUNEL-positive cells were calculated as they represent apoptosis cells. For further confirmation, the activity of caspase- 3 was quantified using a Caspase-3/CPP32 Colorimetric Assay kit (BioVision, Inc.). Caspase-3 is a key in apoptosis, as its activity reflects the intensity of apoptosis. 

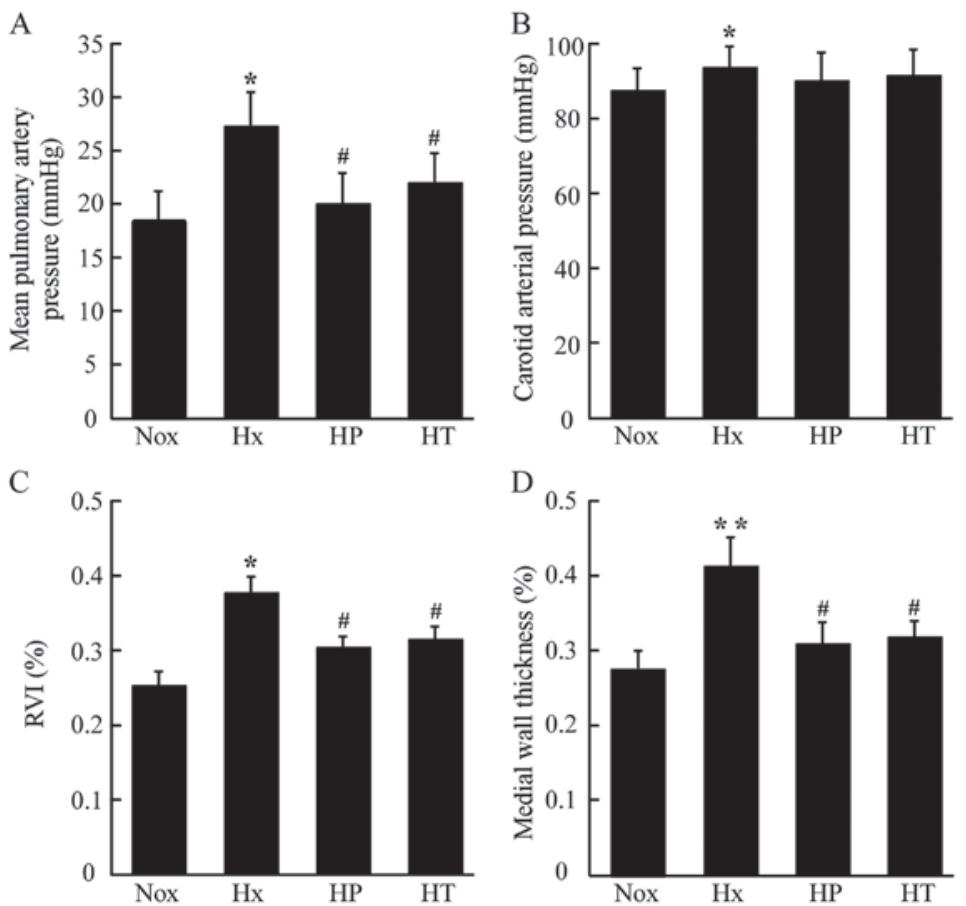

Figure 1. Effect of AS on hypoxia-induced pulmonary hypertension in rats. (A) Mean pulmonary artery pressure. (B) Carotid arterial pressure. (C) Right ventricular hypertrophy $(\mathrm{RV} / \mathrm{LV}+\mathrm{S})$. (D) Medial wall thickness. Data are presented as the mean \pm standard deviation. ${ }^{*} \mathrm{P}<0.05$, ${ }^{* *} \mathrm{P}<0.01$ vs. Nox group; ${ }^{*} \mathrm{P}<0.05$ vs. Hx group, $\mathrm{n}=10$. AS, asiaticoside; Nox, control rats raised in normoxia for 4 weeks; Hx, control rats raised in hypoxia for 4 weeks; HP, rats raised in hypoxia and received AS from the same day for 4 weeks; HT, rats raised in hypoxia for 2 weeks, then received the 4-week AS treatment.

Cytosolic extracts were incubated in 96 -well plates at $37^{\circ} \mathrm{C}$ for 1-2 h with DEVD-pNA substrate $(200 \mu \mathrm{M}$ final concentration). Absorbance was detected in a microtiter plate reader at $400 \mathrm{~nm}$. Results were calculated by the equation obtained from a standard curve.

Western blot analysis. The protein expression of Akt, phosphorylated (p)-Akt at $\mathrm{Ser}^{473}$, eNOS, and p-eNOS at Ser ${ }^{177}$ in lung tissue of rats was detected by western blot analysis. Frozen lung tissue was prepared and homogenized in lysis buffer (Beyotime Institute of Biotechnology, Haimen, China), sonicated twice and then centrifuged for $20 \mathrm{~min}$ at $10,000 \mathrm{x} \mathrm{g}$. The expression of these proteins in HPAECs was also quantified $24 \mathrm{~h}$ after the aforementioned treatments. Cell proteins were isolated from HPAECs by centrifuging at $4^{\circ} \mathrm{C}$ for $5 \mathrm{~min}$ at $12,000 \mathrm{x} \mathrm{g}$ and using lysis buffer (Beyotime Institute of Biotechnology). To determine the protein concentration of the lysate, the Bradford method was used with bovine serum albumin (ScienCell Research Laboratories, Inc., San Diego, CA, USA) as the standard. Proteins from each sample with equal amounts $(25 \mu \mathrm{g})$ were resolved by SDS-PAGE $(12 \%$ separation gel) and then transferred onto polyvinylidene fluoride membranes (EMD Millipore, Billerica, MA, USA). Western blots were blocked by incubating with PBS containing 5\% skimmed milk for $1 \mathrm{~h}$ at room temperature. The membrane was then incubated with the primary antibodies overnight at $4^{\circ} \mathrm{C}$. The primary antibodies used were as follows: Anti-eNOS (cat. no. 5880, 1:1,000; Cell Signaling Technology, Inc., Danvers, MA, USA), anti-p-eNOS (cat. no. 9571, 1:1,000; Santa Cruz Biotechnology, Inc., Dallas, TX, USA), anti-Akt (cat. no. 2920, 1:1,000), anti-p-Akt (cat. no. 4051, 1:1,000) (both from Cell Signaling Technology, Inc.), and anti-GAPDH (cat. no. G8795, 1:1,000; Sigma-Aldrich; Merck Millipore). Subsequently, the blots were incubated with peroxidase conjugated goat anti-rabbit secondary antibody (cat. no. sc-2004, 1:2,000; Santa Cruz Biotechnology, Inc.) for $1 \mathrm{~h}$. Subsequently, peroxidase labeling was visualized via enhanced chemiluminescence reagent provided by Thermo Fisher Scientific, Inc. (Waltham, MA, USA). By scanning the X-ray film (Bio-Rad Laboratories, Inc., Hercules, CA, USA), densitometry results were analyzed using Image-Pro Plus 6.0 (Media Cybernetics, Inc., Rockville, MD, USA) software (National Institutes of Health, Bethesda, MD, USA). All experiments were repeated at least three times.

Statistical analysis. Data are expressed as the mean \pm standard deviation. Between-group mean comparisons were performed using one-way analysis of variance followed by a Student-Newman-Keuls test using $\mathrm{R}$ version 3.3.2. $\mathrm{P}<0.05$ were considered to indicate a statistically significant difference.

\section{Results}

$A S$ inhibits the development of hypoxic $P H$, cardiovascular remodeling and endothelial cell injury in hypoxic $P H$. As presented in Fig. 1A, mPAP in Hx group increased compared with that in the Nox group. AS administered at $50 \mathrm{mg} / \mathrm{kg}$ daily for 4 weeks in prevention and treatment groups (HP group and HT group, respectively) inhibited the elevation of mPAP induced by hypoxia. There was no significant difference between the HP and HT groups. AS seemed to have no impact on the systemic blood pressure, as carotid arterial pressure (CAP), although there was an increased level of CAP in Hx group compared with the Nox group. (Fig. 1B). After 

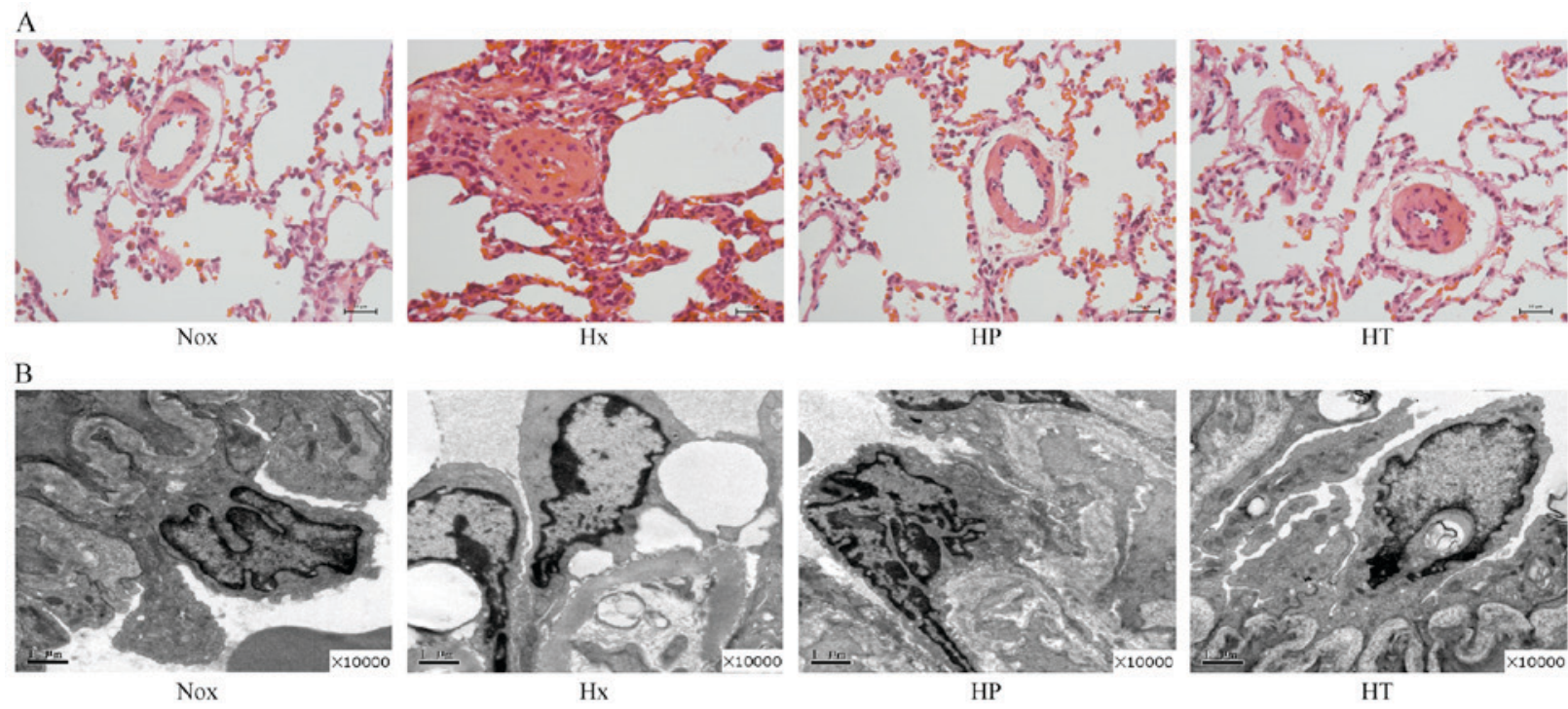

Figure 2. Effect of AS on the morphometry of pulmonary arterioles and epithelial cells in hypoxic pulmonary hypertension rats. (A) Histological features of pulmonary arterioles by hematoxylin and eosin staining. (B) Ultrastructural changes of endothelial cells by transmission electron microscope detection. AS, asiaticoside; Nox, control rats raised in normoxia for 4 weeks; Hx, control rats raised in hypoxia for 4 weeks; HP, rats raised in hypoxia and received AS from the same day for 4 weeks; HT, rats raised in hypoxia for 2 weeks, then received the 4 -week AS treatment.

exposure to hypoxia, rats exhibited more severe right ventricular hypertrophy (RVI=RV/LV+Sep), which was relieved in both the HP and HT groups (Fig. 1C). Analyses of medial wall thickness of pulmonary arterioles revealed that the Hx group had much higher medial wall thickness than the Nox group, whereas the HT groups showed reduced higher medial wall thickness than Hx (Fig. 1D).

Compared with the Nox group, the lumina of pulmonary arterioles in Hx group was reduced, with inflammatory cells were located along vascular walls (Fig. 2A). These abnormalities were partly ameliorated in the HP and HT groups, with the HP group that started treatment at beginning of hypoxia exposure having reduced pulmonary arteriole wall thickening and inflammatory cell infiltration. At ultrastructure level (Fig. 2B), the pulmonary arteries of the Hx group compared with Nox group showed swelling of endothelial, edema of mitochondria, increased vacuoles, destructive cell junction and exfoliation of basement membrane. Additionally, clustered collagen fibers deposited in adventitia pulmonary arterial walls were observed. Conversely, reduced morphological abnormality of endothelial cells was identified in the HP and HT groups.

AS modulates dysregulation of ET-1, PGI2, cGMP and NO in hypoxic PH. The circulating concentration of ET-1 was markedly elevated in the Hx group compared with the Nox group, whereas it was lower in the HP and HT groups compared with the in Hx group (Fig. 3A). The serum level of NO which was represented by nitric products was reduced in the Hx group compared with the Nox group. The level of NO was restored in HP group, whereas no significant difference was identified in the HT group (Fig. 3B). The serum levels of PGI2 showed no significant differences among these groups (Fig. 3C). The cGMP concentrations in lung tissue demonstrated similar pattern as the NO levels in the serum. The HP group had higher cGMP concentration compared with the Hx group, which was significantly reduced compared with Nox group
(Fig. 3D). However, no significant difference was identified between the Hx and HT group.

AS upregulates activation of Akt and eNOS in lung tissue. To examine the effect of AS on the activation of Akt and eNOS in vivo, the present study assessed the expression of Akt and eNOS, and their phosphorylated products, p-Akt at $\mathrm{Ser}^{473}$ and p-eNOS at $\mathrm{Ser}^{1177}$ in lung tissues. The relative ratio of p-Akt/AKT and p-eNOS/eNOS represent the activation of the key proteins in NO-mediated signaling. The phosphorylation of AKT was elevated in the Hx, HP and HT groups. The HP and HT groups had higher levels of activation of AKT compared with the Nox group (Fig. 4A and B). The phosphorylation of eNOS decreased significantly in the Hx group compared with the Nox group. The HP and HT groups were increased in the phosphorylation of eNOS compared with Hx group, whereas the HP group had a higher phosphorylation level compared with the HT group (Fig. 4C and D), which indicated that early treatment of AS contributes to higher phosphorylation levels in hypoxia.

AS prevents endothelial cells from hypoxia-induced inhibition of cell viability and NO production. To evaluate whether AS protects HPAECs from hypoxia-induced damage in vitro, cell viability was quantified using CCK-8 tests (Fig. 5A). A significant increase in absorbance was observed upon treatment with AS under hypoxia exposure in a dose-dependent manner between 12.5 and 50 , and 25 and $50 \mu \mathrm{g} / \mathrm{ml}$ of AS (H25 and H50) compared with control in hypoxia (H0), and their counterparts in normoxia has no significant difference; however, AS (50 $\mu \mathrm{g} / \mathrm{ml})$ together with LY294002 (20 $\mu \mathrm{mol} / \mathrm{l})$ significantly suppressed the cell viability either under normoxia or hypoxia condition. As presented in Fig. 5B, NO secretion was reduced in the $\mathrm{H} 0$ group compared with the Nox group, whereas it was significantly increased in the H50 group compared with the H0 group. Nevertheless, there was 

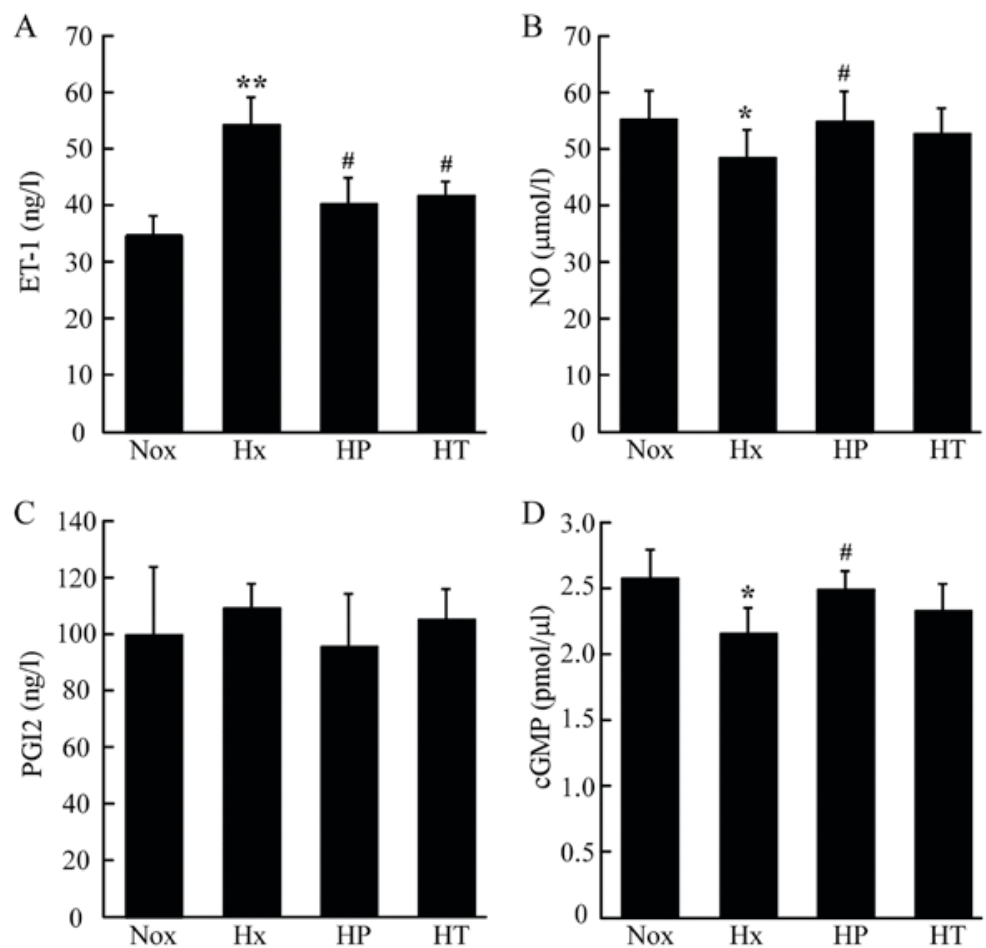

Figure 3. Effect of AS on concentrations of vascular activators in rats tested by ELISA kits. (A) Concentrations of (A) ET-1 (B) NO (C) PGI2 in serum and (D) cGMP in lung tissue. ${ }^{*} \mathrm{P}<0.05,{ }^{* *} \mathrm{P}<0.01$ vs. Nox group; ${ }^{*} \mathrm{P}<0.05$ vs. Hx group, $\mathrm{n}=10$. AS, asiaticoside; ET-1, endothelin-1, NO, nitric oxide; PGI2, prostacyclin; cGMP, cyclic guanosinc monophosphate; Nox, control rats raised in normoxia for 4 weeks; Hx, control rats raised in hypoxia for 4 weeks; HP, rats raised in hypoxia and received AS from the same day for 4 weeks; HT, rats raised in hypoxia for 2 weeks, then received the 4-week AS treatment.
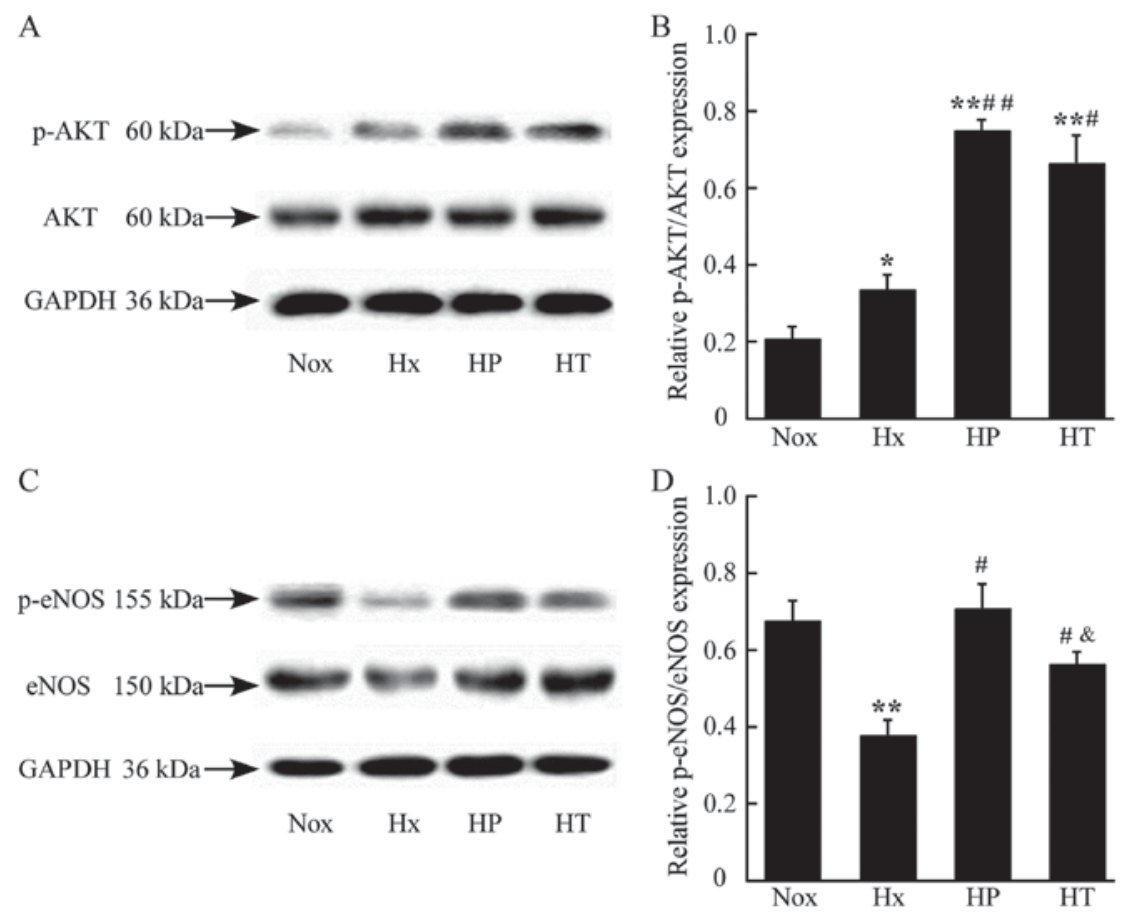

Figure 4. Effect of AS on the activation of eNOS and Akt in lungs of rats assessed by western blotting. (A) Demonstrative immunoblot of Akt and p-Akt in rat lung tissue. (B) Densitometric analysis of expression of p-Akt/Akt ratio. (C) Demonstrative immunoblot of eNOS and p-eNOS in rat lung tissue. (D) Densitometric analysis of expression of $\mathrm{p}-\mathrm{eNOS} / \mathrm{eNOS}$ ratio. ${ }^{\prime} \mathrm{P}<0.05,{ }^{* * *} \mathrm{P}<0.01$ vs. Nox group; ${ }^{\#} \mathrm{P}<0.05,{ }^{\# \#} \mathrm{P}<0.01$ vs. Hx group; ${ }^{\text {\& }} \mathrm{P}<0.05$ vs. HP group, $\mathrm{n}=3$. AS, asiaticoside; Akt, serine/threonine-specific protein kinase; p-Akt, phosphorylated-Akt; eNOS, endothelial nitric oxide synthase; Nox, control rats raised in normoxia for 4 weeks; Hx, control rats raised in hypoxia for 4 weeks; HP, rats raised in hypoxia and received AS from the same day for 4 weeks; HT, rats raised in hypoxia for 2 weeks, then received the 4 -week AS treatment.

a reduced NO secretion in the HL group compared with the Nox or H50 groups.
AS protects endothelial cells from hypoxia-induced apoptosis. A TUNEL assay was used to determine whether AS 

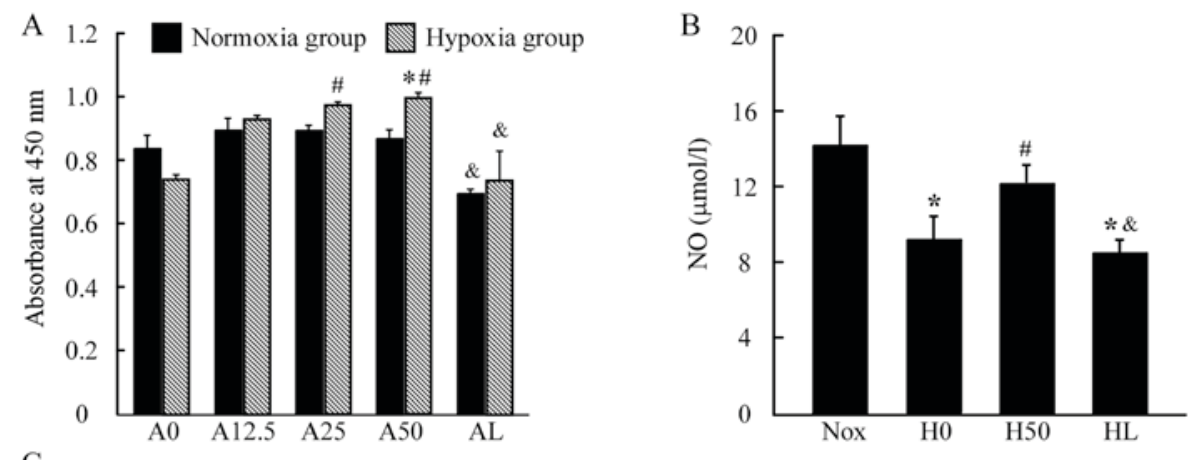
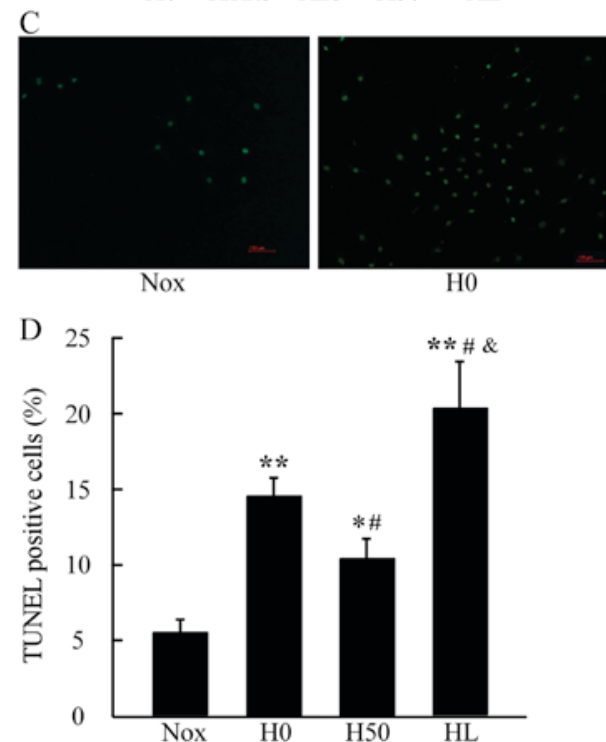
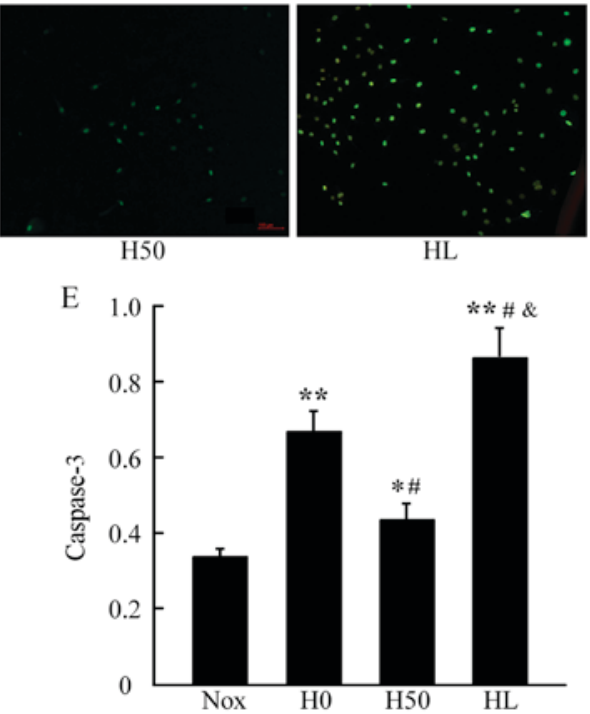

Figure 5. Protective role of AS for HPAECs. (A) Cell viability was determined using Cell Counting Kit-8. (B) NO production in HPAECs. (C and D) TUNEL assay showing the differences of apoptotic rates under specific conditions. (E) Activity of caspase- 3 to support the apoptosis evidence. ${ }^{*}<0.05,{ }^{* *} \mathrm{P}<0.01$ vs. Nox group; ${ }^{\#} \mathrm{P}<0.05$ vs. H0 group; ${ }^{\circledR} \mathrm{P}<0.05$ vs. H50 group, n=3. HPAECs, human pulmonary artery endothelial cells; AS, asiaticoside; NO, nitric oxide; Nox, control rats raised in normoxia for 4 weeks.

protects HPAECs by blocking hypoxia-induced apoptosis (Fig. 5C and D). As illustrated, the H0 group exhibited obvious signs of apoptosis compared with Nox, whereas AS treatment (H50 group) prevented hypoxia-induced apoptosis, restoring cell survival under hypoxic conditions. However, the HL group presented a higher level of cell apoptosis compared with the H0 group. Similar pattern was observed in the activity of caspase- 3 in these groups. Active caspase-3 was noticeably increased in the HO group compared to the Nox group, the H50 group had reduced caspase-3 activity compared with the H0 group, whereas there was significantly increased level of caspase-3 activity in the HL group (Fig. 5E).

AS upregulated and phosphorylation of AKT/eNOS in hypoxia-exposed HPAECs. Western blot analysis was performed to investigate the effect of the AS treatment on the activation of Akt and eNOS in HPAECs in vitro. The hypoxia stimulation of HPAECs induced lower phosphorylation of AKT by detecting the p-AKT/AKT expression ratio, which was significantly increased by AS treatment $(50 \mu \mathrm{g} / \mathrm{ml})$, whereas treatment combined with LY294002 (HL group) markedly reduced the phosphorylation of AKT induced by the AS treatment. Additionally, AS $(50 \mu \mathrm{g} / \mathrm{ml})$ upregulated the phosphorylation of eNOS compared with the hypoxia or Nox group $(\mathrm{P}<0.05)$, whereas LY294002 and
AS combined (HL group) downregulated the phosphorylation of eNOS $(\mathrm{P}<0.05)$. These findings indicated that AS treatment may increase the phosphorylation of AKT/eNOS in hypoxia conditions, whereas LY294002 may inhibit this effect (Fig. 6C and D).

\section{Discussion}

Based on previous observations, the present study determined that in addition to the preventive effect, AS performs a beneficial role in established hypoxic PH as it was evident that AS treatment may restore pulmonary artery pressure without causing systemic hypotension, and attenuate RV hypertrophy, vascular remodeling and ECs morphology changes induced by hypoxia in rats. These findings indicate that AS is a potential option for the prevention and treatment of hypoxic $\mathrm{PH}$.

Endothelial damage is a key initial event in $\mathrm{PH}$. Hypoxia results in the reduction of NO production by interfering the activation of eNOS (20). To the best of our knowledge the present study was the first to demonstrate clearly that AS is capable of maintaining the regular morphology and vital functions of endothelial cells and correcting the NO release in vivo. ET-1 is a potent vasoconstrictor, whereas cGMP has vasodilatory and anti-platelet aggregation properties (21). The disequilibrium of those factors underpin various 

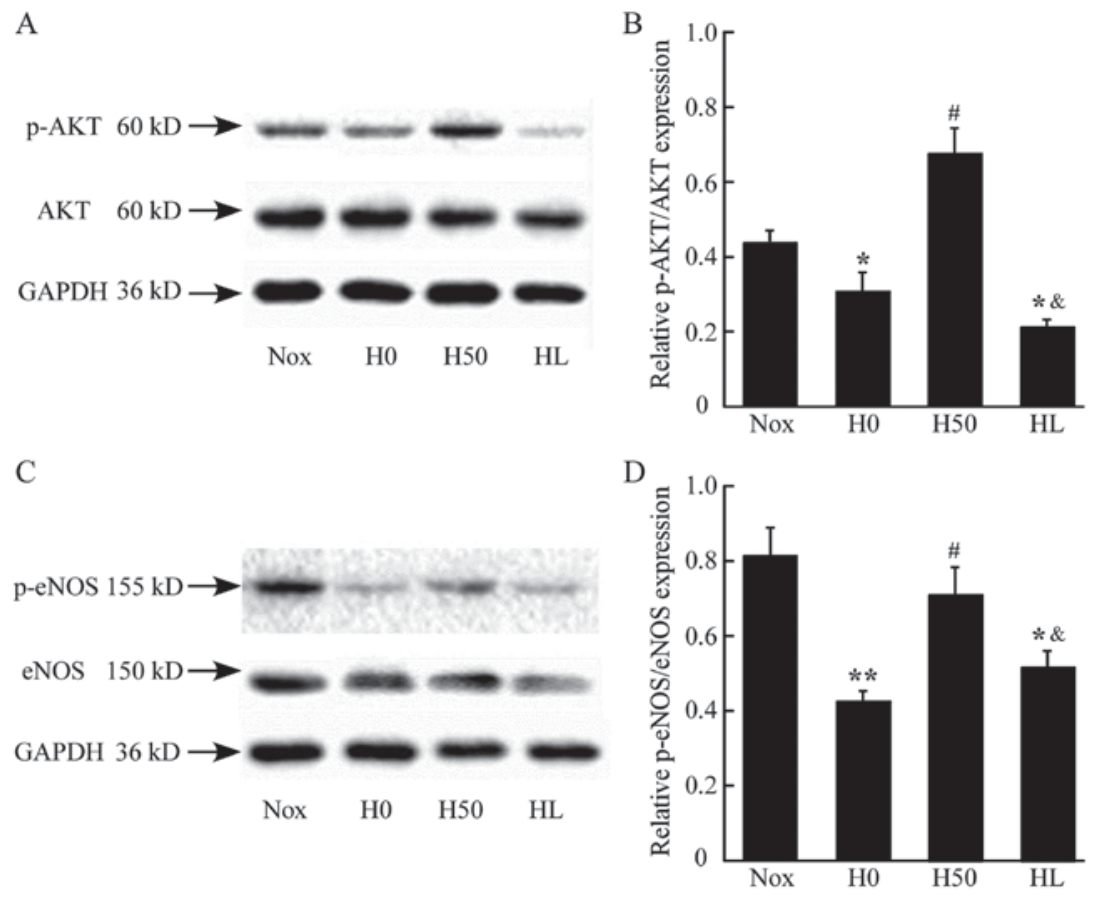

Figure 6. Effect of AS on the activation of eNOS and Akt in HPAECs was assessed by western blotting. (A) Demonstrative immunoblot of Akt and p-Akt in HPAECs. (B) Densitometric analysis of relative expression of p-Akt/Akt ratio. (C) Demonstrative immunoblot of eNOS and p-eNOS in HPAECs. (D) Densitometric analysis of relative expression of $\mathrm{p}$-eNOS/eNOS ratio. ${ }^{*} \mathrm{P}<0.05,{ }^{* *} \mathrm{P}<0.01$ vs. Nox group; ${ }^{\text {}} \mathrm{P}<0.05$ vs. H0 group; ${ }^{\&} \mathrm{P}<0.05$ vs. H50 group, $\mathrm{n}=3$. AS, asiaticoside; HPAECs, human pulmonary artery endothelial cells; Akt, serine/threonine-specific protein kinase; p-Akt, phosphorylated-Akt; eNOS, endothelial nitric oxide synthase.

morphological and hemodynamic changes in PH (22). In addition to the ultrastructural restoration of ECs, misadjusted levels of vascular mediators like ET-1, cGMP and NO were ameliorated following AS treatment. It is of note, that the AS treatment administered 2 weeks after the hypoxia exposure (HT group) had reduced levels of cGMP and NO restoration in vivo, which suggested that early treatment would bring about improved outcomes. Increased NO production by AS was consistent with the significant activated phosphorylation of eNOS at $\operatorname{Ser}^{1177}$. Akt has been determined to activate the phosphorylation of eNOS, inhibit apoptotic processes, thus interfering with cellular survival pathways (23). The upregulation of Akt and eNOS activation by AS treatment in the present study may contribute to the normalization of NO-mediated signal and the preservation of endothelial cell morphology and function, which inhibits the progression of hypoxic PH in rats.

The present study confirmed the role of AS on the PI3K/Akt signaling pathway by the experiments in HPAECs in vitro using a specific PI3K inhibitor, LY294002. The PI3K/Akt signaling pathway has a critical role in cell survival during hypoxia, and phosphorylation of Akt protects cells against hypoxia-induced apoptosis $(24,25)$. The present study determined that hypoxia decreased cell viability and induced apoptosis in HPAECs, which is in accordance with a previous study (26); however, this was significantly reversed by AS $(50 \mu \mathrm{g} / \mathrm{ml})$ treatment, which demonstrated the protective effects on HPAECs. However, the cytoprotective effect of AS was reduced by PI3K inhibitors LY294002 which induced apoptosis and inhibited viability in ECs under hypoxia. LY294002 may also reduce the effect of AS on the activation of AKT and eNOS, which indicates that AS activated eNOS through a PI3K/Akt-dependent mechanism in
HPAECs, which eventually resulted in the reduced generation of NO by ECs. To the best of our knowledge for the first time, the present study demonstrated that AS treatment upregulated the PI3K/Akt/eNOS pathway in vivo and in vitro.

There are limitations of the present study. The rat hypoxic PH model used cannot fully represent the molecular complexity present in human patients. Additionally, AS may enhance NO production via activation of the AKT/eNOS pathway, whereas the precise molecular mechanisms underlying the process and their cause-and-effect relationship remain to be elucidated. Additional studies are required in order to resolve these questions.

In conclusion, the present findings suggest that there are significant effects of AS on preventing and reversing hypoxic $\mathrm{PH}$. The present study revealed that the AS acted to promote $\mathrm{NO}$ production in circulation of a hypoxic $\mathrm{PH}$ rat model, and in HPAECs culture under hypoxia where it had an inhibitory effect on hypoxia-induced apoptosis. The AS-mediated protective effect on ECs was accompanied by phosphorylation of Akt and eNOS in vivo and in vitro. In addition, this protective effect was significantly inhibited by LY294002 treatment in vitro, indicating that PI3K/Akt/eNOS signal pathways have key roles in hypoxic $\mathrm{PH}$ and act as a possible target for AS treatment in hypoxic PH. These findings imply that AS may be a potential therapeutic option for hypoxic $\mathrm{PH}$.

\section{Acknowledgements}

The present study was supported by the National Science Foundation of China (grant nos. 81470250, 81473406 and 81700062), the Natural Science Foundation of Zhejiang 
Province (grant no. LQ16H010003 and LY13H020005) and the Science and Technology Project of Wenzhou (grant no. Y20140048).

\section{References}

1. Rubin LJ: Primary pulmonary hypertension. N Engl J Med 336: 111-117, 1997.

2. Humbert M, Morrell NW, Archer SL, Stenmark KR, MacLean MR, Lang IM, Christman BW, Weir EK, Eickelberg O, Voelkel NF and Rabinovitch M: Cellular and molecular pathobiology of pulmonary arterial hypertension. J Am Coll Cardiol 43 (12 Suppl S): 13S-24S, 2004.

3. Shimoda LA, Sham JS and Sylvester JT: Altered pulmonary vasoreactivity in the chronically hypoxic lung. Physiol Res 49 549-560, 2000.

4. Xu W, Koeck T, Lara AR, Neumann D, DiFilippo FP, Koo M, Janocha AJ, Masri FA, Arroliga AC, Jennings C, et al: Alterations of cellular bioenergetics in pulmonary artery endothelial cells. Proc Natl Acad Sci USA 104: 1342-1347, 2007.

5. Campbell AI, Zhao Y, Sandhu R and Stewart DJ: Cell-based gene transfer of vascular endothelial growth factor attenuates monocrotaline-induced pulmonary hypertension. Circulation 104: 2242-2248, 2001.

6. Zhao YD, Campbell AI, Robb M, Ng D and Stewart DJ: Protective role of angiopoietin-1 in experimental pulmonary hypertension. Circ Res 92: 984-991, 2003.

7. Le Cras TD and McMurtry IF: Nitric oxide production in the hypoxic lung. Am J Physiol Lung Cell Mol Physiol 280: L575-L582, 2001.

8. Fulton D, Gratton JP, McCabe TJ, Fontana J, Fujio Y, Walsh K, Franke TF, Papapetropoulos A and Sessa WC: Regulation of endothelium-derived nitric oxide production by the protein kinase Akt. Nature 399: 597-601, 1999.

9. Ou J, Fontana JT, Ou Z, Jones DW, Ackerman AW, Oldham KT, Yu J, Sessa WC and Pritchard KA Jr: Heat shock protein 90 and tyrosine kinase regulate eNOS $\mathrm{NO}^{*}$ generation but not $\mathrm{NO}^{*}$ bioactivity. Am J Physiol Heart Circ Physiol 286: H561-H569, 2004.

10. Giaid A and Saleh D: Reduced expression of endothelial nitric oxide synthase in the lungs of patients with pulmonary hypertension. N Engl J Med 333: 214-221, 1995.

11. Pullamsetti S, Kiss L, Ghofrani HA, Voswinckel R, Haredza P, Klepetko W, Aigner C, Fink L, Muyal JP, Weissmann N, et al: Increased levels and reduced catabolism of asymmetric and symmetric dimethylarginines in pulmonary hypertension. FASEB J 19: 1175-1177, 2005.

12. Guo JS, Cheng CL and Koo MW: Inhibitory effects of Centella asiatica water extract and asiaticoside on inducible nitric oxide synthase during gastric ulcer healing in rats. Planta Med 70: 1150-1154, 2004.

13. Yun KJ, Kim JY, Kim JB, Lee KW, Jeong SY, Park HJ, Jung HJ, Cho YW, Yun K and Lee KT: Inhibition of LPS-induced NO and PGE2 production by asiatic acid via NF-kappa B inactivation in RAW 264.7 macrophages: Possible involvement of the IKK and MAPK pathways. Int Immunopharmacol 8: 431-441, 2008.
14. Dong MS, Jung SH, Kim HJ, Kim JR, Zhao LX, Lee ES, Lee EJ, Yi JB, Lee N, Cho YB, et al: Structure-related cytotoxicity and anti-hepatofibric effect of asiatic acid derivatives in rat hepatic stellate cell-line, HSC-T6. Arch Pharm Res 27: 512-517, 2004.

15. Chen S, Yin ZJ, Jiang C, Ma ZQ, Fu Q, Qu R and Ma SP: Asiaticoside attenuates memory impairment induced by transient cerebral ischemia-reperfusion in mice through anti-inflammatory mechanism. Pharmacol Biochem Behav 122: 7-15, 2014.

16. Wang XB, Wang W, Zhu XC, Ye WJ, Cai H, Wu PL, Huang XY and Wang LX: The potential of asiaticoside for TGF- $\beta 1 /$ Smad signaling inhibition in prevention and progression of hypoxia-induced pulmonary hypertension. Life Sci 137: 56-64, 2015.

17. Muramatsu M, Oka M, Morio Y, Soma S, Takahashi H and Fukuchi Y: Chronic hypoxia augments endothelin-B receptor-mediated vasodilation in isolated perfused rat lungs. Am J Physiol 276: L358-L364, 1999.

18. Michelakis ED, McMurtry MS, Wu XC, Dyck JR, Moudgil R, Hopkins TA, Lopaschuk GD, Puttagunta L, Waite R and Archer SL: Dichloroacetate, a metabolic modulator, prevents and reverses chronic hypoxic pulmonary hypertension in rats: Role of increased expression and activity of voltage-gated potassium channels. Circulation 105: 244-250, 2002.

19. Zheng Y, Li M, Zhang Y, Shi X, Li L and Jin M: The effects and mechanisms of mycophenolate mofetil on pulmonary arterial hypertension in rats. Rheumatol Int 30: 341-348, 2010.

20. Mukhopadhyay S, Xu F and Sehgal PB: Aberrant cytoplasmic sequestration of eNOS in endothelial cells after monocrotaline, hypoxia and senescence: Live-cell caveolar and cytoplasmic NO imaging. Am J Physiol Heart Circ Physiol 292: H1373-H1389, 2007.

21. Wilkins MR: Pulmonary hypertension: The science behind the disease spectrum. Eur Respir Rev 21: 19-26, 2012.

22. Coggins MP and Bloch KD: Nitric oxide in the pulmonary vasculature. Arterioscler Thromb Vasc Biol 27: 1877-1885, 2007.

23. Song G, Ouyang G and Bao S: The activation of Akt/PKB signaling pathway and cell survival. J Cell Mol Med 9: 59-71, 2005.

24. Mangi AA, Noiseux N, Kong D, He H, Rezvani M, Ingwall JS and Dzau VJ: Mesenchymal stem cells modified with Akt prevent remodeling and restore performance of infarcted hearts. Nat Med 9: 1195-1201, 2003.

25. Martorell L, Gentile M, Rius J, Rodríguez C, Crespo J, Badimon L and Martínez-González J: The hypoxia-inducible factor 1/NOR-1 axis regulates the survival response of endothelial cells to hypoxia. Mol Cell Biol 29: 5828-5842, 2009.

26. Pullamsetti SS, Savai R, Janssen W, Dahal BK, Seeger W, Grimminger F, Ghofrani HA, Weissmann N and Schermuly RT: Inflammation, immunological reaction and role of infection in pulmonary hypertension. Clin Microbiol Infect 17: 7-14, 2011.

This work is licensed under a Creative Commons Attribution-NonCommercial-NoDerivatives 4.0 International (CC BY-NC-ND 4.0) License. 